\title{
Linx
}

Revue des linguistes de l'université Paris X Nanterre

$7 \mid 1995$

Saussure aujourd'hui

\section{Saussure 'héraclitéen' : épistémologie constructiviste et réflexivité de la théorie linguistique}

René Amacker

\section{OpenEdition}

Journals

Édition électronique

URL : http://journals.openedition.org/linx/1122

DOI : 10.4000/linx.1122

ISSN : 2118-9692

Éditeur

Presses universitaires de Paris Nanterre

Édition imprimée

Date de publication : 1 septembre 1995

Pagination : 17-28

ISSN : 0246-8743

Référence électronique

René Amacker, «Saussure 'héraclitéen' : épistémologie constructiviste et réflexivité de la théorie linguistique », Linx [En ligne], 7 | 1995, mis en ligne le 12 juillet 2012, consulté le 10 décembre 2020. URL : http://journals.openedition.org/linx/1122 ; DOI : https://doi.org/10.4000/linx.1122

Ce document a été généré automatiquement le 10 décembre 2020.

Département de Sciences du langage, Université Paris Ouest 


\title{
Saussure 'héraclitéen' : épistémologie constructiviste et réflexivité de la théorie linguistique
}

\author{
René Amacker
}

\section{A Alexandra Alexandrovna A.}

1 À propos du «facteur Temps » et de l'identification problématique des états de langue, Saussure a noté, en vue d'une de ses conférences de novembre 1891, ces mots caractéristiques :

[1] [...] il n'arrive jamais qu'une langue succède à une autre; par exemple que le français succède au latin; [...] cette succession imaginaire de deux choses vient uniquement de ce qu'il nous plaît de donner deux noms successifs au même idiome, et par conséquent d'en faire arbitrairement deux choses séparées dans le temps. Sans doute, l'influence qu'exercent sur notre esprit deux noms successifs de ce genre est tellement décisive et tellement inébranlable, indéracinable, que je ne songe pas, je vous l'avoue franchement, à détruire votre préjugé en quelques jours par deux ou trois remarques de ma part $(\mathrm{N} 1.3=3285,2){ }^{1}$

Ces lignes sont tirées d'un développement méthodologique où figurent les principaux éléments du problème épistémologique dont il va être question ici: d'une part, l'arbitraire de l'observateur, c'est-à-dire du linguiste («il nous plaît [...] d'en faire arbitrairement deux choses séparées dans le temps »), et surtout, d'autre part, l'influence de la langue sur qui se sert de la langue, c'est-à-dire notamment sur le théoricien de la langue (« l'influence qu'exercent sur notre esprit deux noms successifs de ce genre est [...] décisive et [...] inébranlable »).

2 Ce dernier point résulte de la réflexivité foncière et irréductible de la langue. La réflexivité, qui se traduit dans la langue par des propriétés particulières, ${ }^{2}$ parait requérir une épistémologie elle aussi particulière, dans les termes de laquelle il soit possible de comprendre les propriétés que la langue doit à la réflexivité. Saussure, apparemment sensible à ces exigences épistémologiques singulières, a ressenti de manière très vive, et pour ainsi dire tragique, les conséquences paradoxales qu'elles entraînaient dans la 
pratique de sa discipline. C'est ainsi, par sa conscience d'avoir élaboré une conception de la langue qu'alors personne ne partageait, que s'expliqueraient, à mon sens, à la fois la solitude scientifique de Saussure, telle ou telle des déclarations dramatiques que j'aurai l'occasion de répéter, et, par voie de conséquence, ce que l'on a appelé le 'silence' qui a caractérisé ses années genevoises.

\section{Réflexivité}

3 Avant d'entrer en matière, il me faut préciser ce que j'entends par réflexivité et quelle est la conséquence qu'elle entraîne au point de vue épistémologique.

4 La réflexivité est la propriété qu'a toute langue naturelle d'être sa propre métalangue : on a besoin de la langue pour parler de la langue, ce qui signifie que les énoncés théoriques du linguiste s'expriment au moyen de cela même précisément sur quoi ils portent. Autrement dit, quelle que soit la propriété que le linguiste reconnaît à la langue, cette propriété appartient en principe aux énoncés qui formulent la propriété en question. Ainsi, affirmer l'arbitraire radical du signe linguistique, c'est du même coup affirmer l'arbitraire radical du signe arbitraire radical. ${ }^{3}$

5 La réflexivité linguistique ne se confond pas avec le cercle herméneutique; car le linguiste qui se sert de la langue pour décrire la langue ne cherche pas à enrichir, à chacun de ses retours sur l'objet de sa description, sa propre connaissance de l'objet, mais, partant de sa connaissance de sa langue (connaissance intuitive et, en ce qui le concerne lui-même en ce moment précis, connaissance complète), il se propose d'établir une construction formelle de l'objet de sa science qui soit telle que, en s'enrichissant progressivement (c'est-à-dire en devenant progressivement plus complexe), elle finisse par coïncider avec la langue de la description, fournissant non seulement l'interprétation des faits de langue ${ }^{4}$ mais surtout - par réflexivité - les moyens formels précisément qui auront été utilisés pour établir cette construction même.

6 Si j'ai réussi à faire saisir ce que j'ai voulu dire, la langue devrait apparaître, en tant qu'elle est réflexive, comme un ruban de Mœbius du premier type (tel que celui qui s'y promène peut changer de face du ruban sans changer de face du ruban $)^{5}$ ou, mieux encore, comme le moulin du tableau d'Escher, dont l'eau qui par sa chute vient de faire tourner la roue revient, en courant tranquillement dans son chenal, faire tourner la roue par sa chute. Tout se passe donc comme si la langue réalisait le mouvement perpétuel. ${ }^{6}$

7 Je voudrais montrer ici que la réflexivité de la langue et de la linguistique a hanté Saussure, comme on s'en rend compte à lire des textes théoriques, d'une part, et des textes d'ordre général, d'autre part. Mais pour commencer, il me faut encore m'expliquer sur l'usage de l'adjectif 'héraclitéen' et sur la mention de l'épistémologie constructiviste dans mon titre.

\section{Constructivisme 'héraclitéen'}

8 Il y a évidemment quelque anachronisme à parler d'épistémologie constructiviste à propos de Saussure. Pourtant, s'il s'avère que le Genevois a cherché à maîtriser dans sa théorie les effets de la réflexivité, la linguistique saussurienne doit être foncièrement constructiviste (au sens que Piaget a donné à ce terme). ${ }^{7}$ 
Le constructivisme de Saussure se manifeste de diverses manières, notamment par le rôle de la psychologie dans la théorie, ${ }^{8}$ et surtout par le passage « de la théorie du signe aux termes du système " (titre de Godel 1966). Je ne répéterai pas ce que j’ai écrit jadis du constructivisme tel qu'il se manifeste à chacun des deux niveaux, sémiologique et proprement linguistique (cf. Amacker 1975 : 99-102). Et quelques mots suffiront à rappeler que j'ai tenté naguère d'utiliser, aussi rigoureusement que possible, le constructivisme saussurien pour mettre au point une méthode de description syntaxique fondée sur la notion de dépendance, qui devait s'appliquer au latin (Amacker 1986, chapitre I). La leçon principale que j'ai tirée de cet essai, c'est qu'une telle entreprise, quelque soin qu'on mette à respecter la cohérence de la construction, réclame presque à chaque pas des décisions ad hoc laissées - certes dans des limites étroites - à la libre appréciation du linguiste (d'ailleurs le choix même des éléments initiaux - en particulier la notion de dépendance, dans mon cas - est déjà lui aussi laissé à la libre appréciation du linguiste). ${ }^{9}$ Il s'agit là d'un aspect de ce que j'appelle l'arbitraire du grammairien, et que je mets à présent au compte de la réflexivité.

Si le constructivisme de Saussure mérite à mon sens d'être dit 'héraclitéen', c'est pour une série de raisons, dont la première est que la langue est en devenir constant : l'objet du linguiste incarne par excellence la thèse maîtresse de l'Ephésien. On peut d'ailleurs entendre, dans certaines des expressions que Saussure utilise, un écho reconnaissable des formules d'Héraclite.

11 Ainsi, dans une note autographe qui correspond à une leçon du troisième cours, Saussure a-t-il insisté très fortement sur le mouvement de la langue dans le temps : «Il n'y a pas d'exemple d'immobilité absolue. Ce qui est absolu, c'est le principe du mouvement de la langue dans le temps » (N 23.1 p. $7=2205 \mathrm{~F})$, texte dans lequel on peut voir une variante $\mathrm{du}$ principe héraclitéen le plus connu, selon lequel 'tout coule', qui donne contradictoirement lui aussi - une forme absolue à ce qui doit se concevoir comme radicalement changeant.

12 Le même texte de Saussure se poursuit par une allusion probable à Héraclite : "On ne verra jamais que le fleuve de la langue soit identique [...]; il suffit qu'il existe imperturbablement, naturellement, et au-dessus de toute circonstance " (ibid.). Pour insister sur le devenir universel, Héraclite a justement pris le fleuve comme exemple (cf. en particulier les fragments 16 et 52 Diano-Serra); ainsi, dans la formulation traditionnelle, 'on ne se baigne jamais deux fois dans le même fleuve'. ${ }^{10}$

13 S'il ne s'agissait que de la coïncidence des métaphores, on ne pourrait pas en tirer de conclusion spéciale. Mais l'héraclitisme de Saussure se manifeste encore, et bien plus sérieusement, à propos de l'identité linguistique. Dans ses notes pour un article sur Whitney, l'écho de la célèbre formule d'Héraclite me paraît indéniable : «L'objet qui sert de signe [c'est-à-dire le son, la réalisation du signifiant (RA)] n'est jamais 'le même' deux fois » $(\mathrm{N} 10,1=3297){ }^{11}$

\section{La réflexivité dans la linguistique}

14 Cette version 'héraclitéenne' du constructivisme est, pour Saussure, le produit, tout à la fois, de la réflexion épistémologique sur le langage, et de la nature même de la langue. ${ }^{12}$ En tout cas, c'est ainsi que j'interprète la thématique du 'point de vue', qui concerne en premier lieu l'objet de la linguistique : 
[2] L'objet qui sert de signe n'est jamais 'le même' deux fois ; il faut dès le premier moment un examen ou une convention initiale pour savoir au nom de quoi, dans quelles limites nous avons le droit de l'appeler le même; là est la fondamentale différence avec un objet quelconque [...] (N 10,1 = 3297).

Mais en second lieu, par réaction de l'objet sur la description de l'objet (de la nature du premier sur les propriétés de la seconde), la thématique du 'point de vue' affecte la théorie linguistique elle-même. La découverte que la linguistique elle-même est soumise à l'arbitraire du 'point de vue', parce que c'est la langue qui sert à exposer la théorie, a vivement frappé Saussure, qui en a vu les conséquences paradoxales. La première et celle qu'il a le plus nettement soulignée, c'est que la langue est un objet « sans analogue » $:^{13}$

[3] [...] le langage et l'écriture ne sont PAS FONDÉS sur un rapport naturel des choses.

[...] C'est ce que Whitney ne s'est jamais lassé de répéter pour mieux faire sentir que le langage est une institution pure. Seulement cela prouve beaucoup plus, à savoir que le langage est une institution sans analogue (si l'on y joint l'écriture) [...] $(\mathrm{N} 10,18=1264 \mathrm{D}) \mathbf{.}^{14}$

Un peu plus avant dans la même note, on trouve la même idée, exprimée avec une emphase qui trahit l'importance que Saussure lui accorde :

[4] « Nous sommes [...] profondément convaincu que quiconque pose le pied sur le terrain de la langue peut se dire qu'il est abandonné par toutes les analogies du ciel et de la terre. C'est précisément pourquoi on a pu faire sur la langue d'aussi fantaisistes constructions que celles que démolit Whitney [...] $»(\mathrm{~N} 10,38=1267 \mathrm{~F})$.

Ces textes ne font pas directement allusion à la réflexivité. ${ }^{15}$ Néanmoins, si je les verse au présent dossier, c'est parce que Saussure, à un ensemble de considérations relatives aux propriétés de la langue (ici, à son caractère "sans analogue »), en mêle d'autres qui concernent les propriétés de la linguistique. De fait - deuxième conséquence remarquable - à mesure que l'arbitraire radical de la langue se révèle à Saussure, c'est l'arbitraire de la linguistique qui le hante davantage. La terminologie, notamment, en est comme minée de l'intérieur ; qui plus est, cette instabilité foncière au niveau théorique se répercute au niveau épistémologique. En 1894, Saussure écrit :

[5] Il serait par suite impossible [...] de discourir sur un seul des termes usités en linguistique dans la pratique de chaque jour sans reprendre ab ovo la question totale du langage $(\mathrm{N} 10=3297,33)$.

Quelques années plus tard, il reconnaît clairement que les difficultés terminologiques contre lesquelles il se bat sont l'effet de la réflexivité :

[6] Même un terme comme sôme [...] deviendrait en très peu de temps, s'il avait la chance d'être adopté, synonyme de sème, auquel il veut être opposé. C'est ici que la terminologie linguistique paie son tribut à la vérité même que nous établissons comme fait d'observation (note Item 3318.8).

La formule - qui doit être postérieure à 1897 (Godel 1957 : 37) - mérite d'être mise en évidence, et méditée. ${ }^{16}$

Saussure avait d'ailleurs déjà dit la même chose, mais de façon plus sibylline, dans la conférence de 1891 déjà citée :

[7] Il y aura un jour un livre spécial et très intéressant à écrire sur le rôle du mot comme principal perturbateur de la science des mots $(\mathrm{N} 1.3=3285,8) .{ }^{17}$

Dans la perspective épistémologique du constructivisme 'héraclitéen', j'accorde à la réinterprétation (qu'on appelle parfois 'métanalyse') une importance centrale ; en effet, ce phénomène est à mon sens une des conséquences les plus apparentes de la réflexivité. Dans la langue, il se manifeste notamment dans ce que Sechehaye (probablement à la suite de ses entretiens avec Saussure) a décrit comme l'attribution, par le sujet parlant, de sa propre grammaire à la parole de son interlocuteur $:^{18}$ 
[8] Une phrase construite grammaticalement d'après une certaine règle, est analysée d'après une règle nouvelle (Sechehaye $1908: 172$ ).

Un peu plus haut, décrivant le même phénomène comme moteur du changement diachronique, Sechehaye avait été plus précis :

[9] Pour attribuer à un signe une valeur grammaticale, [...] il n'est pas nécessaire que ce symbole existe déjà dans ma grammaire à moi. Je crée en attribuant à celui que j'entends, la grammaire dont je crois reconnaître les éléments dans sa parole $:^{19}$ c'est un phénomène d'intuition, et cette grammaire, qui au fond est de mon invention, je l'adopte pour moi-même en me basant sur l'autorité de la personne à laquelle je l'ai gratuitement attribuée (Sechehaye 1908 : 165).

18 De même, concernant du moins la pratique de Saussure, la réinterprétation - cette fois celle qui se manifeste dans l'activité du linguiste - peut être une clé herméneutique fructueuse ; je pense bien sûr au cas extrême de la recherche sur les anagrammes (dont les règles méthodologiques et même l'objet changent, comme on le sait, à mesure qu'elle se développe), ${ }^{20}$ mais il y en a d'autres exemples. Le plus frappant peut-être se trouve dans la question des unités: nécessairement conçues comme segmentales pour commencer, elles deviennent tout aussi nécessairement 'abstraites' quand on applique jusqu'au bout l'analyse segmentale. ${ }^{21}$

Un autre exemple de réinterprétation (ici, potentielle) figure dans le passage suivant, qui concerne la morphologie :

[10] L'idée que pour voir ce qui est au fond des formes, il n'y a que 'd'analyser ces formes', comme on analyse une substance chimique ou comme on dissèque, recouvre un monde de naïvetés et de conceptions étonnantes. C'est montrer que ( $1^{\circ}$ ) il y a vingt sortes d'analyses n'ayant rien de commun et n'ayant une valeur que si on les a classées, $\left(2^{\circ}\right)$ l'objet n'est pas analysable avant d'avoir une existence définie (N $12=3299,25 ;$ c'est moi qui souligne).

Enfin, plus caractéristiques encore, ces deux textes, que séparent sans doute plus de quinze ans :

[11] Il faudrait, pour présenter convenablement l'ensemble de nos positions, adopter un point de départ fixe et défini. Mais tout ce que nous tendons à établir, c'est qu'il est faux d'admettre en linguistique un seul fait comme défini en soi. Il y a donc véritablement absence nécessaire de tout point de départ, et si quelque lecteur veut bien suivre attentivement notre pensée, d'un bout à l'autre de ce volume, il reconnaîtra, nous en sommes persuadé, qu'il était pour ainsi dire impossible de suivre un ordre très rigoureux. Nous nous permettrons de remettre jusqu'à trois et quatre fois, sous différentes formes, la même idée sous les yeux du lecteur, parce qu'il n'existe réellement aucun point de départ plus indiqué qu'un autre pour y fonder la démonstration $(\mathrm{N} 9.1,4-5=128 \mathrm{~F})$.

A ces lignes rédigées avant 1894, et qui prennent tout leur sens quand on les interprète dans l'optique de la réflexivité, correspondent les mots notés par Léopold Gautier le 6 mai 1911, où réinterprétation et réflexivité apparaissent, encore plus nettement que dans le texte [11], comme des propriétés de la formalisation de la linguistique :

[12] Pour le moment, la linguistique générale m'apparaît comme un système de géométrie. On aboutit à des théorèmes qu'il faut démontrer. Or on constate que le théorème 12 est, sous une autre forme, le même que le théorème 33 («Entretien Gautier », dans Godel 1957 : 30).

21 Tout ce que j'ai essayé de dire jusqu'ici se trouve dans ces quelques phrases: conformément aux exigences du constructivisme, la position de Saussure est provisoire (cf. "pour le moment»); la linguistique est un système formel (cf. [12] "système de géométrie ", « démontrer », [11] « démonstration »); la réinterprétation aboutit à reconnaître l'identité de «théorèmes » d'abord différents ([12]), c'est-à-dire à « remettre 
[...] sous des formes différentes la même idée sous les yeux du lecteur » ([11]); et elle est la contrepartie de la réflexivité, qui s'exprime (a) dans le fait que Saussure veut "établir " formellement (cf. « nécessaire ») qu'il n'y a pas un « point de départ » « défini en soi » sur lequel «fonder la démonstration» formelle ([11]), et (b), dans le fait qu'on assiste, au cours de la construction du "système ", à un changement des points de vue définissant les différents « théorèmes » ([12]).

\section{En guise de conclusion}

Si la langue est un objet "sans analogue », c'est aussi qu'il s'agit d'une forme particulière de connaissance, dont la science est par définition une épistémologie, et l'épistémologie, une épistémologie d'épistémologie. Saussure ne pouvait disposer d'aucun moyen technique pour formaliser ni la réflexivité ni ce nœud conceptuel unique. Pourtant, il ne s'est pas borné à observer les effets " perturbateurs ", dans la science de la langue, de la réflexivité ou de la singularité radicale de la langue; les textes que j'ai cités montrent qu'il a cherché les causes des perturbations observées, et qu'il les a trouvées précisément dans la réflexivité. ${ }^{22}$ Par ailleurs, il ne fait de doute pour personne que le fond de la pensée saussurienne est d'ordre éminemment épistémologique ; il suffira de citer deux textes bien connus :

[13] On ne peut se refuser, avant tout, à reconnaître que la théorie du langage aura pour plus essentielle tâche de démêler ce qu'il en est de nos distinctions premières (N 9.1,1 = 1977 F [date : avant 1894]).

[14] Préoccupé surtout depuis longtemps de la classification logique de ces faits [les «faits de langage»(RA)], de la classification des points de vue sous lesquels nous les traitons, je vois de plus en plus [...] l'immensité du travail qu'il faudrait pour montrer au linguiste ce qu'il fait, en réduisant chaque opération à sa catégorie prévue (« Lettre à Meillet », dans Godel $1957: 31$ [date : janvier 1894]). ${ }^{23}$

Comme il n'est pas question de reprendre ici le dossier de Saussure épistémologue, je me contenterai d'une brève suggestion, qui devrait rendre vraisemblable par un autre biais encore la thèse que je défends. Voici : à mon avis, c'est en partant de ce qu'il tirait de l'observation de la langue - c'est-à-dire des lois ou des « théorèmes » de la linguistique que Saussure en est venu à postuler le cadre pour ainsi dire vide qu'est chez lui la sémiologie; c'est que la sémiologie, une fois qu'elle aurait été constituée, devait simplement fonder et déterminer la linguistique, c'est-à-dire justement le point de départ de Saussure. Il me semble donc que, selon une reconstruction idéale de sa démarche, la sémiologie, définie au moyen des instruments conceptuels fournis par la linguistique (à un niveau $N$ de la formalisation), était conçue en particulier pour fournir les instruments conceptuels (de niveau $\mathrm{N}-1$ dans la formalisation) propres à définir formellement, parmi d'autres disciplines de niveau $\mathrm{N}$, notamment la linguistique. ${ }^{24} \mathrm{~J}$ 'attribue donc à un effet de la réflexivité la raison d'être de la sémiologie, qui ne sert visiblement à rien d'autre chez Saussure qu'à donner à la linguistique une caution scientifique dont la linguistique assure elle-même le financement.

Si ces vues ne sont pas totalement chimériques, on conçoit que la sémiologie saussurienne en soit restée pour l'essentiel à l'état de projet: les moyens, notamment formels, qui auraient été indispensables à son développement échappaient à Saussure; mais le seul fait qu'il en a postulé l'existence prouve, à mon sens, que son épistémologie était constructiviste. 
On conçoit aussi que Saussure ait éprouvé très vivement sa solitude de savant, et qu'il se soit déclaré en désaccord radical avec toutes les écoles :

[15] Du moment qu'il ne s'agit plus que des choses universelles qu'on peut dire sur le langage, je ne me sens d'accord avec aucune école en général, pas plus avec la doctrine raisonnable de Whitney qu'avec les doctrines déraisonnables qu'il a victorieusement combattues. [...] Et ce désaccord est tel qu'il ne comprend aucune transaction ni nuance, sous peine de me voir obligé d'écrire des choses n'ayant aucun sens à mes yeux (N 10 = 3297,23-23a).

On conçoit aussi ses scrupules de professeur :

[16] Je me trouve placé devant un dilemme : ou bien exposer le sujet dans toute sa complexité et avouer tous mes doutes, ce qui ne peut convenir pour un cours qui doit être matière à examen. Ou bien faire quelque chose de simplifié, mieux adapté à un auditoire d'étudiants qui ne sont pas linguistes. Mais à chaque pas, je me trouve arrêté par des scrupules (« Entretien Gautier », dans Godel $1957: 30$ ).

On conçoit aussi le caractère aphoristique de sa pensée, et les formules si frappantes qui expriment tout à la fois sa solitude et le caractère à peine pensable de ce qu'il pense. ${ }^{25}$ C'est ainsi en tout cas que je m'explique non seulement l'emphase du texte [4], mais surtout - à propos de la valeur linguistique - la mention d'un 'désespoir de l'esprit' dans une note de 1911 :

[17] Prenant la chose échangeable d'une part, de l'autre les termes cosystématiques, cela n'offre aucune parenté. C'est le propre de la valeur de mettre en rapport ces deux choses. Elle les met en rapport d'une manière qui va jusqu'à désespérer l'esprit par l'impossibilité de scruter si ces deux faces de la valeur diffèrent pour elle ou en quoi $(\mathrm{N} 23.6,11=1864 \mathrm{~F})$.

Enfin, maintenant que les raisons scientifiques et non pas seulement personnelles de son 'silence' nous sont devenues intelligibles, on conçoit qu'il n'ait rien voulu publier : poussé par la contrainte de la langue (réalité mouvante et « sans analogue » qui longtemps parut lui échapper dans la mesure même où il pensait la saisir), Saussure s'est engagé sur un terrain épistémologique qui se dérobe sous vos pieds dès que vous y prenez pied.

Un mot pour finir. Tout ce que nous tirons des notes autographes et même des notes des cours de linguistique générale est foncièrement fragmentaire; comme dans le cas d'Héraclite, les sources que nous lisons et interprétons sont évidemment insuffisantes à nous représenter pleinement l'état et l'évolution de la pensée saussurienne: les notes autographes indépendantes des cours sont anciennes et disparates; quant aux cours, Saussure n'a pas pu leur donner la forme qui eût convenu à l'état de sa réflexion (cf. texte [16]). Ainsi avons-nous été à jamais privés des idées que, fascinés nous-mêmes par l'épistémologie 'héraclitéenne' qu'il nous enseigne, nous cherchons chez lui, sans que nous puissions être assurés de les y trouver jamais : car seule une " méditation exclusive " de plusieurs mois, comme il le confiait à Léopold Gautier, lui aurait permis de leur donner forme, voire simplement de les faire exister. ${ }^{26}$

\section{BIBLIOGRAPHIE}

René Amacker, Linguistique saussurienne, Droz, Genève, 1975. 
René Amacker, Structures et conventions, Albert Meynier, Turin, 1986.

René Amacker, « La creatività del ricevente secondo Albert Sechehaye », dans T. De Mauro, S. Gensini, M. E. Piemontese (a c. di), Dalla parte del ricevente : Percezione, comprensione, interpretazione (Atti del XIX Congresso Internazionale, Roma, 8-10 novembre 1985), Bulzoni, Roma, 1988, p. 61-71.

Eugenio Coseriu, « Principes de syntaxe fonctionnelle », Travaux de linguistique et de philologie, Strasbourg-Nancy, 1989, p. 5-46.

Carlo Diano \& Giuseppe Serra, Eraclito. I frammenti e le testimonianze, Fond. L. Valla, Mondadori, s.l., ${ }^{2} 1987\left({ }^{1} 1980\right)$.

Rudolf Engler : v. Saussure.

Robert Godel, Les sources manuscrites du Cours de linguistique générale de F. de Saussure, Droz, Genève, 1957.

Robert Godel, « De la théorie du signe aux termes du système », Cahiers Ferdinand de Saussure 22, 1966, p. 53-68.

André Martinet, Syntaxe générale, Armand Colin, Paris, 1985.

Jean-Claude Milner, «La théorie syntaxique peut-elle analyser ses propres énoncés ? , Cahiers Ferdinand de Saussure 43, 1989, p. 7-21.

Jean Piaget, « Les courants de l'épistémologie scientifique contemporaine », dans J. Piaget, éd., Logique et connaissance scientifique, Encyclopédie de la Pléiade, Gallimard, Paris, 1967, p. 1225-1271.

Ferdinand de Saussure, Cours de linguistique générale. Edition critique par Rudolf Engler, tome 1, Harrassowitz, Wiesbaden, 1968; Id., tome 2, fascicule 4, Harrassowitz, Wiesbaden, 1974.

Albert Sechehaye, Programme et méthodes de la linguistique théorique. Psychologie du langage, Champion, Paris \& Harrassowitz, Leipzig \& Eggimann, Genève, 1908.

Raffaele Simone, Il sogno di Saussure. Otto studi di storia delle idee linguistiche, Laterza, Roma-Bari, 1992.

Jean Starobinski, Les mots sous les mots. Les anagrammes de Ferdinand de Saussure, Gallimard, Paris, 1971.

\section{NOTES}

1. Les notes de Saussure sont toutes citées d'après l'édition de Rudolf Engler.

2. Par exemple les difficultés liées à la différence entre 'usage' et 'mention' dans les énoncés du théoricien de la syntaxe (cf. Milner 1989).

3. Le paradoxe du menteur est une illustration particulière de cette propriété (exemple mentionné fugitivement par Milner [1989: 8]). - Je cite plus bas (note 16) un exemple indiscutable de réflexivité chez Saussure.

4. Sur le caractère à son tour problématique de la notion de 'fait' linguistique, cf. Simone 1992 : $\mathrm{x}$ xi.

5. F. Lo Piparo a récemment utilisé de manière différente l'analogie du ruban de Moebius pour illustrer un problème de sémiologie générale («Le signe linguistique est-il à deux faces? Saussure et la topologie», communication présentée au colloque "Saussure et la linguistique contemporaine " de Tokyo ; le texte en a paru dans les Cahiers Ferdinand de Saussure 45, 1991, p. 213-221). 
6. Ce n'est évidemment pas dans ce sens que Saussure a parlé de la langue « comparable à une machine qui marcherait toujours, quelles que soient les détériorations qu'on lui ferait subir » (1444 B et E).

7. Du point de vue de l'épistémologie générale, le constructivisme s'oppose à la fois à l'empirisme mécaniste et à l'idéalisme innéiste (cf. notamment Piaget 1967).

8. Cf. mon article «La théorie linguistique de Saussure et la psychologie », Cahiers Ferdinand de Saussure 48, 1994.

9. Dans le cas particulier, j'ai repris et développé à mon usage la méthode qu'enseignait Henri Frei. Mais on sait bien que le structuralisme d'inspiration saussurienne a proposé plusieurs méthodes d'analyse syntaxique, telles la syntaxe générale d'André Martinet (1985) ou la syntaxe fonctionnelle d'Eugenio Coseriu (1989).

10. La formulation varie, car le fragment est attesté par des sources indirectes diverses (cf. Diano-Serra 1980: 154-155); le fragment 52 dit précisément: "Le fleuve dans lequel 㯺les hommes国 entrent est le même, mais les eaux qui courent vers eux sont toujours autres. »

11. Autre rencontre de Saussure avec Héraclite, le rôle de la 'masse parlante', de la collectivité, en particulier pour l'établissement du sens : «Ceux qui parlent de façon sensée doivent trouver leur force dans ce qui est commun à tous, comme la cité la trouve dans la loi » (frg. 8 D-S).

12. Cf. ce texte, qui montre bien la double source que je signale : « Réflexions sur les entités. [...] Compte des causes qui font du langage un objet situé hors de toute comparaison et non classé ni dans l'esprit des linguistes ni dans l'esprit des philosophes » (N $17=3326)$.

13. Saussure réussira à briser l'isolement de la langue grâce à l'invention de la sémiologie (la mention des 'institutions humaines' montre implicitement quel sera le développement futur de ses réflexions).

14. Cf. encore N $1.1=3283,23 ;$ N 10,17 = 1265-1266 E. - Toute la thématique de la langue 'sans analogue' se déploie dans le cadre de la lutte contre les métaphores des linguistes, dont la conclusion paradoxale est qu' « on ne peut ni se passer de ces images, ni se résoudre à les accepter », ce qui est pour Saussure une « cause de dégoût » (N $10=3297,33)$.

15. Le texte [4] mentionne toutefois la pluralité des " constructions » théoriques, qui se rapporte à la thématique de la réinterprétation, elle-même effet de la réflexivité (cf. plus bas).

16. On trouve un exemple précis de réflexivité sous la plume de Saussure: "Il n'y a aucun substratum quelconque aux entités linguistiques; elles ont la propriétés d'exister de par leur différence, sans que le pronom elles [dans la phrase en cours, qui commence par «elles ont la propriété »(RA)] arrive où que ce soit à désigner autre chose lui-même qu'une différence » $(\mathrm{N} 24 \mathrm{a}=3342.2,1)$.

17. Cf. ce texte qui établit clairement le lien entre la linguistique et la langue : «Pour confondre l'état et l'événement, la linguistique a des ressources inépuisables. La confusion est dans les idées, mais quand elle ne serait que dans les mots, dans la façon dont nous sommes obligés d'exprimer les faits [ ]» $(\mathrm{N} 12=3299,27)$; on peut imaginer de compléter le texte par : '..., elle ne laisserait pas d'être aussi pernicieuse'.

18. Cf. mon article de 1988.

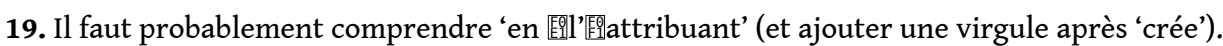

20. Cf. notamment les textes réunis par Starobinski 1971.

21. A quelques jours de distance, Saussure insiste d'abord sur le caractère nécessairement segmental des unités : «Ce qui est essentiel, c'est le problème des unités. En effet, la langue est nécessairement comparable à une ligne dont les éléments sont coupés aux ciseaux, pan, pan, pan [...] » («Entretien Gautier », du 6 mai 1911, dans Godel 1957 : 30 ; cf. «tranche de sonorité [...] liée à un certain concept qui sert à délimiter la tranche ", définition de l'entité concrète [1734 C, de la leçon du 9 mai]), puis se trouve contraint de reconnaître des 'entités abstraites', qui ne répondent assurément plus à la définition de 'tranche', tout en la supposant néanmoins : «Ce 
domaine est un des plus obscurs [...]. Ce sera toujours un domaine qui supposera [Fill'[ilétude préalable des entités concrètes (2166 s. B[E], de la même leçon [cf. Godel 1957 : 83]).

22. Aux textes [6] et [7], on ajoutera encore cette phrase, qui établit implicitement un rapport d'identité entre une propriété de l'exposé théorique du linguiste et une propriété de l'objet de cet exposé : «L'idée que les choses de la langue doivent pouvoir s'exposer par une voie une et suivie est la même idée fausse qui fait qu'on suppose que la langue elle-même est une chose une » (N $12=3299,17)$.

23. Dans la même lettre, Saussure insiste sur «cette ineptie de la terminologie courante, la nécessité de la réformer, et de montrer pour cela quelle espèce d'objet est la langue en général »; pour «éviter les expressions logiquement odieuses », « il faudrait [...] une réforme décidément radicale »: on ne saurait mieux marquer le lien qu'il y a entre la nature particulière de l'objet 'langue' et la science qui le décrit.

24. $\mathrm{Si}$, à cause de la réflexivité (dont ce serait là une des conséquences les plus graves), la langue doit foncièrement se dérober à une authentique formalisation logico-mathématique, on comprendra ici 'formalisation' au sens de 'formalisation faible' (cf. Amacker 1975 : 11-12) ou de 'quasi-formalisation'.

25. Il est piquant de rappeler qu'Héraclite avait apparemment donné à son oeuvre la forme de sentences ou d'aphorismes (cf. H. Dörrie, art. « Herakleitos », dans Der Kleine Pauly, Bd. 2 [1975], 1046).

26. Je tiens à remercier ici Giorgio Quadranti, à qui je dois et l'idée de cette communication et ce que j'ai pu comprendre du mécanisme formel de la réflexivité.

\section{AUTEUR}

\section{RENÉ AMACKER}

(Université de Genève) 